\title{
MANEJO ODONTOLÓGICO DE PACIENTES EM TERAPIA ANTITROMBÓTICA
}

\author{
DENTAL MANAGEMENT OF PATIENTS ON \\ ANTITHROMBOTIC THERAPY
}

\begin{abstract}
Andreza Pereira Gonçalves de Souza*, Francyelle Deodato Cavalcante*, Eteíla de Souza Canto Silva**, Suely Fernandes Santana**, Marcílio Otávio Brandão Peixoto***, Fernanda Braga Peixoto***
\end{abstract}

Autora para correspondência: Andreza Pereira Gonçalves de Souza - andrezapgs@gmail.com

*Graduanda do Curso de Odontologia do Centro Universitário Cesmac, Maceió, Alagoas

**Graduada do Curso de Odontologia do Centro Universitário Cesmac, Maceió, Alagoas

***Mestre em Ensino na Saúde e Professor do Curso de Odontologia do Centro Universitário Cesmac, Maceió, Alagoas

\section{R E S U M O}

\begin{abstract}
A terapêutica com implantes dentários osseointegráveis tem sido utilizada em todo o mundo, entretanto, revela complicações biológicas, como as alterações peri-implantares que podem levar à perda dos mesmos. Essas alterações podem ser classificadas em mucosite peri-implantar e peri-implantite. Existem múltiplos fatores associados com as alterações peri-implantares, dentre estes, o diabetes mellitus (DM). O DM é um grupo de alterações metabólicas, caracterizada pela hiperglicemia crônica resultante de defeitos na secreção ou ação da insulina, podendo ser DM tipo 1 ou DM tipo 2. Apresenta influência na saúde peri-implantar já que é responsável por aumento da inflamação, estresse oxidativo e apoptoses celulares com consequente retardo na cicatrização. A peri-implantite é a maior responsável pela perda de implantes dentários, por isso, conhecer a relação do DM no processo da doença é fundamental para criação de protocolos de prevenção, tratamento e manutenção da saúde peri-implantar. $\bigcirc$ objetivo deste trabalho é relatar o caso de um paciente com obesidade e diabetes mellitus tipo 2 , reabilitado com 4 implantes osseointegráveis em região posterior e bilateral de mandíbula nos anos de 2008/2009 que, após 5 anos, apresentou peri-implantite nas unidades reabilitadas com considerável perda óssea.
\end{abstract}

Palavras-chave: Diabetes Mellitus; Peri-Implantite; Osseointegração 
The cardiovascular diseases are common and represent the cause of deaths in the world. According to World Health Organization, it is estimated that those are responsible for about 17, 3 million or $30 \%$ of total deaths in the world each year. Among cardiovascular diseases, the thrombotic disease is one of the most prevalent. Pharmaceutical strategies to the thrombosis treatment, due to the predominance of platelet and fibrin depending on the type of thrombus, include anti-platelets medicine, anticoagulant and fibrinolitical agents, even though they have different courses of action, they aim to interfere on main steps of forming and maintenance of blood clots, increasing substantially the risk of spontaneous or induced bleedings. The general trend is upward for patients who are being treated with oral anticogulant to be able to undergo dentistry procedures, with no need of any interruption or modification during therapy, yet with emphasis on preventive procedures of local hemostasis. This approach is still controversial to patients which make use of AAS, considering that the action of interrupting the usage some days before the procedure continues to be a prudent course of action in order to reduce the risk of hemorrhage, and differently from anticoagulants, apparently with no damages to the patient. This study focused on describing the procedures to be adopted for a safe dentistry assistance for patients undergoing anticoagulant, antiplatelet or thrombolytic therapy, based on literature review, using books, articles and scientific journals published in electronical databases such as SciELO, MedLine and Lilacs, in English and Portuguese, besides available information in official electronical addresses such as the Brazilian Ministry of Health's and the World Health Organization's.

Keywords: Thrombolytic therapy. Platelet aggregation inhibitors. Odontology. 


\section{INTRODUÇÃO}

O termo hemostasia significa prevenção da perda sanguínea'. Neste processo há interrupção do sangramento de vasos sanguíneos lesados tornandose, portanto, um fenômeno essencial à manutenção da vida. O processo envolve desde alterações vasculares até a formação de um coágulo de fibrina ${ }^{2}$.

Evolutivamente os seres humanos desenvolveram um sistema bem regulado de hemostasia para manter - sangue no estado fluido e livre de coágulos nos vasos normais e para formar rapidamente um coágulo localizado em áreas de lesão vascular ${ }^{3}$.

Denomina-se como trombose a ativação patológica do processo hemostático dentro de um vaso na ausência de sangramento. Tal distúrbio, que apresenta variação na história natural e morfologia, é frequentemente classificado de acordo com sua localização em arterial ou venoso ${ }^{4}$.

Existem possibilidades de altíssima morbidade e mortalidade quando ocorre a formação ou liberação de trombos de seus sítios locais, pois estes formam êmbolos que flutuam pela circulação e geram consequências à distância, levando à necessidade de tratamento médico contínuo de uma grande quantidade de pessoas acometidas por tal problema $^{2,5}$.

As doenças cardiovasculares são comuns e representam a principal causa de mortes no mundo 6 . Segundo a Organização Mundial da Saúde (OMS) ${ }^{7}$, estima-se que estas são responsáveis por cerca de 17,3 milhões ou $30 \%$ do total mundial de mortes a cada ano, sendo que, desproporcionalmente, mais de $80 \%$ dessas mortes ocorrem em países de baixa e média renda. Dentre as doenças cardiovasculares a doença trombótica é uma das mais prevalentes ${ }^{2}$.

As estratégias farmacêuticas para o tratamento da trombose, devido à predominância de plaquetas e fibrina a depender do tipo de trombo, incluem fármacos antiplaquetários, anticoagulantes e agentes fibrinolíticos, que apesar de possuírem mecanismos de ação distintos, visam interferir em etapas principais da formação e manutenção do coágulo, aumentando significativamente $\mathrm{o}$ risco de sangramento espontâneos ou provocados ${ }^{2,8}$.

Os anticoagulantes orais e injetáveis, bem como os antiagregantes plaquetários, podem ser utilizados como medida profilática ou terapêutica. Já os vários fármacos fibrinolíticos são usados principalmente para reabrir artérias ocluídas de pacientes internos em ambiente hospitalar ${ }^{9,10}$.

Na prática clínica, as indicações de terapêutica antitrombótica vêm se tornando cada vez mais frequentes, devido ao envelhecimento progressivo da população e associado aos maus hábitos alimentares, tabagismo, sedentarismo e excesso de peso, que são considerados os principais fatores de risco para as doenças cardiovasculares ${ }^{11,12}$.

Estima-se que mais de $30 \%$ da população acima dos 70 anos apresenta indicação desta terapêutica, consequentemente aumentando a ocorrência de pacientes usuários deste tipo de medicação nos consultórios odontológicos ${ }^{12,13} \circ$ que pode resultar na ocorrência de hemorragias como acidente ou complicação pós-operatória' ${ }^{14}$.

Para evitar esta temível situação, a realização de procedimentos de forma segura deve estar diretamente relacionada à análise da amplitude do trauma cirúrgico do procedimento proposto com - risco hemorrágico para o paciente usuário de fármacos antitrombóticos ${ }^{12}$.

No entanto, ainda existem muitas dúvidas com relação à suspensão, manutenção ou alteração na terapia antitrombótica de pacientes que serão submetidos a procedimentos odontológicos. Às vezes, a tomada de decisão entre a suspensão ou não da medicação é baseada não em evidências científicas, mas em mitos ${ }^{15}$.

A conduta dos cirurgiões-dentistas em solicitar do médico a suspensão da medicação sem a correta análise dos fatores de risco, deixando a cargo exclusivo deste único profissional qual melhor conduta adotar, pode resultar em maior insegurança para o paciente.

Portanto, o cirurgião-dentista necessita ter acesso a um material de fácil consulta e que reúna informações atuais a respeito deste assunto visando à elaboração de um plano de tratamento adequado 
a depender de cada caso.

Este trabalho objetivou descrever as condutas que devem ser adotadas para $\circ$ atendimento odontológico seguro de pacientes em terapia anticoagulante, antiplaquetária ou trombolítica.

\section{MÉTODO}

Este estudo foi realizado por meio de uma revisão narrativa da literatura, utilizando-se livros, artigos, teses e periódicos científicos publicados em bases eletrônicas como SciELO, Bireme, MedLine e Lilacs, a partir da utilização dos seguintes unitermos: "anticoagulantes", "terapia trombolítica", "inibidores da agregação de plaquetas", "odontologia", "assistência odontológica para idosos", "assistência odontológica para doentes crônicos", nos idiomas português e inglês, além de informações disponibilizadas em endereços eletrônicos oficiais como Ministério da Saúde e OMS.

\section{REVISÃO DE LITERATURA}

O sistema circulatório tem como função suprir as necessidades dos tecidos corporais levando até eles nutrientes, hormônios, eliminando produtos do metabolismo e de maneira geral, mantendo - ambiente apropriado para que as células do organismo possam sobreviver e funcionar adequadamente'.

O coração como a "bomba propulsora" deste sistema participa do funcionamento coordenado junto com os vasos sanguíneos e o sistema linfático4. Em média, o fluxo sanguíneo total na circulação de um adulto é de aproximadamente $5.000 \mathrm{~mL} / \mathrm{min}$. Este flui nos vasos em estado líquido e de forma laminar, ou seja, quando fazendo parte de um sistema estável por um vaso longo e uniforme, organiza-se em linhas de corrente, com as camadas celulares equidistantes da parede do vaso resultando em uma porção de sangue mais central, com o intuito de que essas células centralizadas, na maioria das vezes, não entrem em contato com a camada endotelial ${ }^{16}$.

Também pode ocorrer o oposto com fluxo turbulento onde há sangue correndo em todas as direções e se misturando no interior do vaso, porém nestes casos tende a ocorrer prejuízos ao sistema. Na presença de fluxo turbulento ou de lesões vasculares é que se inicia o processo hemostático?.

A hemostasia é um processo fisiológico envolvido com a fluidez do sangue e com o controle do sangramento quando ocorre lesão vascular, a fim de se evitar a perda sanguínea, tornando-se, portanto, um fenômeno essencial a sobrevivência ${ }^{2,3}$.

Em condições normais, esse processo ocorre pela ação integrada de três fases fundamentais: a fase vascular, a fase plaquetária e da coagulação sanguínea $^{17}$. Penildon Silva ${ }^{10}$ acrescenta ainda uma quarta fase ou fase fibrinolítica ao processo hemostático. As quatro fases da hemostasia e as principais características de cada uma destas podem ser observadas resumidamente na Figura 1, a seguir. 


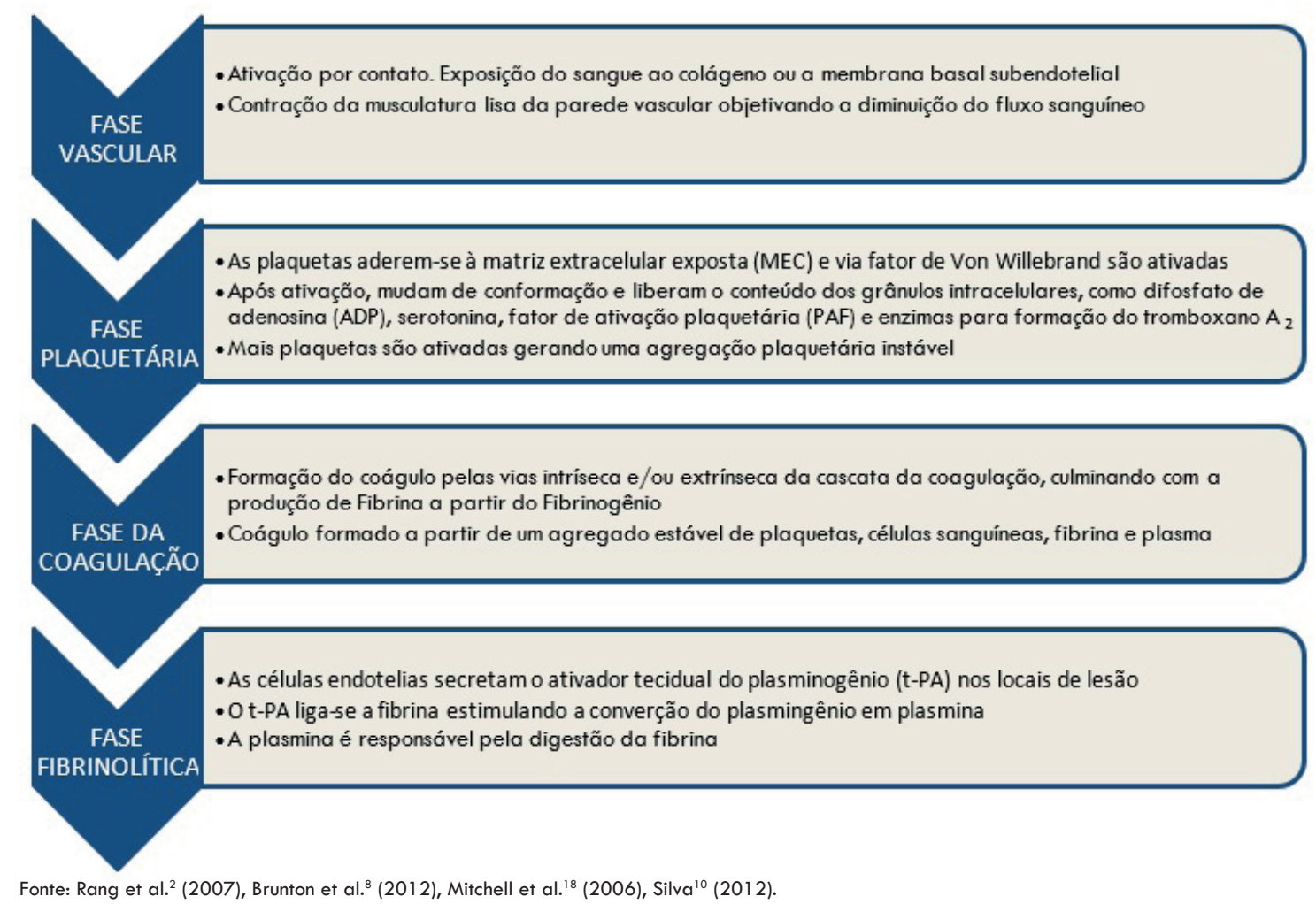

Figura 1. Fases e características do processo hemostático em seres humanos.

Na fase vascular, imediatamente após a lesão, há uma resposta hemostática característica, a vasoconstrição, causada pela contração da musculatura lisa da parede vascular, diminuindo instantaneamente o fluxo de sangue no vaso lesado. É decorrente principalmente do reflexo nervoso desencadeado pelos estímulos gerados pela sensação dolorosa e aumentada pela endotelina, um potente vasoconstritor derivado do endotélio ${ }^{18}$.

As plaquetas desempenham importante papel na hemostasia. Após a lesão vascular estas se aderem à matriz extracelular exposta (MEC) e via fator de von Willebrand presente na parede endotelial lesada são ativadas, mudando de conformação e liberando o conteúdo dos seus grânulos intracelulares, como o difosfato de adenosina (ADP), serotonina, fator de ativação plaquetária (PAF) e as enzimas necessárias para a formação do tromboxano $\mathrm{A} 2$ ? .

A síntese de tromboxano A2 se inicia a partir da formação do ácido araquidônico e é o principal produto da ciclo-oxigenase- 1 envolvido na ativação plaquetária. $O A D P$ e $\circ$ tromboxano A2 atuam nas plaquetas vizinhas ativando-as e aumentando sua adesividade resultando em uma agregação plaquetáriaadicional ou primária ${ }^{10,18}$.
Desta forma, as áreas de soluções de continuidade na parede vascular ativam um número sucessivamente maior de plaquetas com atração mútua, gerando um tampão plaquetário. A princípio esse agregado plaquetário é instável, e só consegue suprimir a perda sanguínea em áreas de pequenas lesões. A seguir, e durante o processo de coagulação do sangue, torna-se compacto devido aos filamentos de fibrina que se prendem formando um tampão hemostático secundário e definitivo ${ }^{19}$.

O terceiro mecanismo da hemostasia é a transformação do sangue líquido na forma de um coágulo gelatinoso. O tempo estimado para - desenvolvimento do coágulo varia de acordo com a gravidade do trauma, ocorrendo dentro de segundos em casos mais graves e em até dois minutos em casos menos severos ${ }^{18}$.

A coagulação sanguínea é resultado de uma sequência de reações em cascata, com participação de mais de uma dúzia de fatores da coagulação sanguínea, onde a cada etapa um fator torna-se uma protease ativa, gerando maior quantidade do fator seguinte, culminando na formação da fibrina insolúvel presente no tampão hemostático secundário. 
Tradicionalmente, a coagulação sanguínea é dividida em vias extrínseca e intrínseca ${ }^{2}$.

A via intrínseca acontece quando há um trauma ao próprio sangue ou o contato do sangue ao colágeno da parede vascular traumatizada, gerando a perda local da característica fisiológica do fluxo sanguíneo laminar. É classicamente iniciada pelo fator XII ou fator de Hageman?.

A via extrínseca ocorre devido à destruição celular, ruptura da parede vascular ou tecidos adjacentes e do contato dos componentes endoteliais expostos com o sangue. Tem início quando o fator III, tecidual ou tromboplastina exposto ativa o fator VII. Então a combinação do fator tecidual com $\circ$ fator VII da coagulação, em presença de íons cálcio, atua enzimaticamente sobre o Fator $X$, viabilizando sua ativação ${ }^{4}$.

Quando da união dessas duas vias, há ativação do Fator $X$, iniciando uma via denominada comum, que culmina com a produção de trombina a partir da protrombina, que é a enzima que viabiliza a conversão do fibrinogênio em polímeros insolúveis de fibrina. O fator XIII ou fator estabilizador da fibrina ativado converte a fibrina em um polímero de ligações cruzadas que fortalece e estabiliza $\circ$ coágulo. $O$ coágulo é resultado de um emaranhado de plaquetas, células sanguíneas, fibrina e plasma ${ }^{17}$.

A figura 2, a seguir, representa esquematicamente a fase de coagulação.

\section{VIA EXTRÍNSECA \\ Trauma tecidual}

Trauma sanguineo
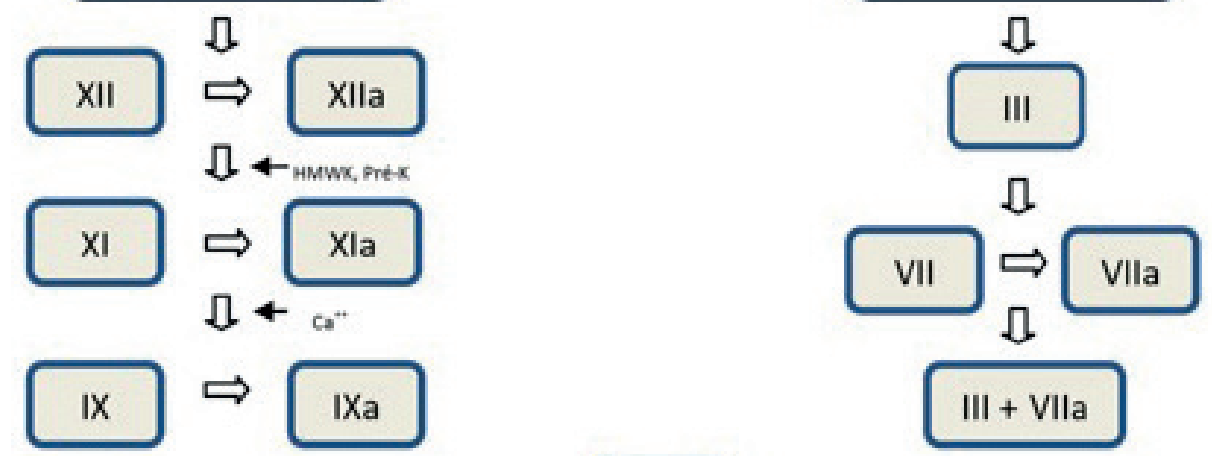

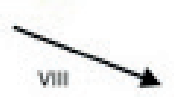

$\mathrm{X}$
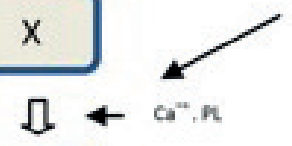

Xa

$\sqrt{2}$
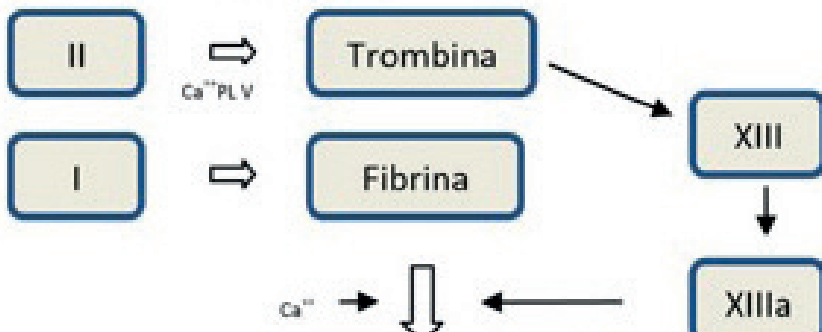

Coágulo

Legenda: XII- Fator de Hageman, a- ativado; HMWK- Fator de Fitzgerald, Pré-K- Fator de Fletcher; XI- Antecedente da tromboplastina plasmática; Ca++- íon cálcio; IX- Componente da tromboplastina plasmática; VIII- Fator anti-hemofílico; III- Fator Tecidual; VIIProconvertina; PL- Fosfolipídios plaquetários; X- Fator de Stuart Prower; II- Protrombina; V- Fator lábil; I- Fibrinogênio; XIII- Fator estabilizador da fibrina.

Fonte: Guyton e Hall' (2006), Silverthorn et al. ${ }^{9}(2010)$.

Figura 2. Cascata da coagulação. 
A cascata da coagulação possui um grande potencial de amplificação, o que justifica a existência primordial de fatores moduladores. Esses fatores moduladores são sistemas anticoagulantes eficazes e capazes de impedir a coagulação exagerada e consequentemente catastrófica para o organismo. O início da coagulação, a ativação dos sistemas anticoagulantes e ao mesmo tempo a fibrinólise são eventos que se sucedem rapidamente ${ }^{4}$.

Uma vez ativada a coagulação, dois mecanismos a restringem ao local da lesão impedindo que continue e promova a coagulação de toda árvore vascular. Primeiro, através da inibição da adesão plaquetária pela liberação de prostaciclina do epitélio vascular íntegro, que impedem a adesão de mais plaquetas, restringindo a formação do tampão plaquetário somente na área lesada e, segundo, pela inibição da cascata da coagulação e consequentemente da produção de fibrina pela proteína $C$ ativada e pela presença de antitrombina $\mathrm{III}^{10,18}$.

Para iniciar a fibrinólise, os ativadores teciduais do plasminogênio (t-PA) convertem o plasminogênio em plasmina, responsável final para dissolução da fibrina presente no coágulo. O sistema fibrinolítico é regulado de modo que os trombos desnecessários de fibrina sejam removidos, enquanto que a fibrina nas áreas de lesão seja conservada para manutenção da hemostasia ${ }^{8}$.

O sistema hemostático quando desregulado pode gerar danos ao sistema vascular, e a sua ativação inapropriada está associada a importantes patologias cardiovasculares ${ }^{17}$.

De acordo com o Ministério da Saúde "1 "as doenças cardiovasculares são as principais causas de morte no Brasil". Entre os anos de 1996 e 2009, a taxa de mortalidade decorrente destas, em todo o país, apresentou tendência ao declínio. No estado de Alagoas, assim como na região nordeste, verificouse o oposto, havendo aumento no número de óbitos no mesmo período. Teixeira et al. ${ }^{5}$ consideram ainda que as enfermidades cardiovasculares são uma das principais causas de mortalidade na sociedade contemporânea, tanto para homens quanto para mulheres.

Tais doenças não são apenas importantes devido ao grande índice de mortalidade, mas também devido à grande quantidade de pacientes afetados e em tratamento médico contínuo. Os principais fatores de risco para doenças cardiovasculares estão associados aos maus hábitos alimentares, tabagismo, sedentarismo e excesso de peso ${ }^{5,11}$.

Dentre as doenças cardiovasculares, a doença trombótica é uma das mais prevalentes ${ }^{2}$. Entende-se por trombose uma ativação inapropriada do sistema hemostático na vasculatura lesionada ou a oclusão trombótica de um vaso após lesão relativamente menor ${ }^{18}$. Caracteriza-se por um processo patológico onde ocorre a solidificação do sangue dentro dos vasos ou do coração de um indivíduo vivo ${ }^{4}$.

A gênese dos fenômenos tromboembólicos ocorre devido a três fatores determinantes, também chamados de Tríade de Virchow. O primeiro fator está relacionado a alterações na parede vascular ou endotelial. É resultado da exposição da matriz extracelular subendotelial, do fator tecidual e da adesão plaquetária ${ }^{19,20}$.

segundo fator refere-se a alterações no fluxo sanguíneo normal, ou seja, fisiologicamente durante - fluxo sanguíneo laminar, os elementos celulares fluem na parte central do lúmen do vaso, sem contato com o endotélio devido a uma camada de plasma $^{1,9}$.

Durante estase ou fluxo turbulento há um rompimento do fluxo laminar levando as plaquetas em contato com o endotélio, impedindo a diluição dos fatores coagulantes ativados e promovendo a ativação celular endotelial $^{18}$.

Por último, as alterações nos constituintes do sangue, por exemplo, em condições que aumentam o número de plaquetas na circulação, contribuem com menos frequência para os estados trombóticos, sem deixar de ser um importante componente ${ }^{19}$.

Algumas condições de hipercoagulabilidade podem determinar um alto risco de trombose, como por exemplo, repouso ou imobilização prolongada no leito, infarto do miocárdio, fibrilação atrial, valvas cardíacas protéticas e danos teciduais diversos, como cirurgias, queimaduras extensas e fraturas ${ }^{18}$.

Os trombos podem se desenvolver em qualquer lugar da circulação. Os de origem arterial ou trombos 
brancos são, em geral, não-oclusivos devido ao fluxo sanguíneo rápido e sob alta pressão nas artérias. Podem ser oclusivos em casos de artérias menores. Constituem-se principalmente de plaquetas e fibrina, entremeadas de hemácias possuindo aspecto seco e friável ${ }^{4,19}$.

Os trombos vermelhos ou venosos são úmidos, gelatinosos e se assemelham ao coágulo sanguíneo. Morfologicamente, cria com frequência um molde longo do lúmen venular, devido à característica estática da circulação venosa. Esses trombos contêm mais hemácias misturadas entre as faixas de fibrina $^{18,19}$.

Os trombos são significativos, pois eles podem causar obstrução de artérias e veias e são fontes de possíveis êmbolos. Entende-se por embolia $\circ$ transporte de fragmentos de um trombo, gordura, gases ou outros corpos estranhos a pontos distantes de sua sede de origem pela corrente sanguínea. Estatisticamente, 99\% das embolias correspondem a deslocamento de trombos, denominando-se assim tromboembolismo ${ }^{19}$.

O tromboembolismo pulmonar é a forma mais comum e das mais graves, uma vez que, não raramente leva o paciente a óbito. É pouco conhecida pela população, porém responsável por milhares de mortes todos os anos. A incidência de trombose venosa profunda e embolia pulmonar é considerada alta no Brasil20. Nos Estados Unidos há uma incidência de 20 a 25 por 100.000 pacientes hospitalizados e cerca de 200.000 mortes a cada ano ${ }^{18}$.

Os êmbolos pulmonares originam-se em cerca de $95 \%$ dos casos de trombos de veias das pernas. A consequência depende basicamente das dimensões do êmbolo e do estado da circulação. De forma habitual, os grandes êmbolos podem alojar-se em diferentes locais e causam morte súbita. Nos de tamanho médio não há formações de lesões significativas em indivíduos saudáveis e os pequenos podem ser menos graves, mas em grande quantidade podem também comprometer a circulação pulmonar ${ }^{18,19}$.

No tromboembolismo arterial, observam-se com frequência êmbolos em artérias do cérebro, miocárdio, extremidades inferiores, baço e rins. Os êmbolos originam-se principalmente em trombos das cavidades esquerdas do coração ${ }^{4}$.

O infarto do miocárdio tem na maioria das vezes a tromboembolia como fator etiológico, mas podem ocorrer devido a outros fatores. Caracteriza-se como uma área localizada de necrose isquêmica, decorrente da falta de aporte sanguíneo numa determinada região de um tecido ou órgão. Em muitos casos ocorre devido à oclusão arterial, podendo em alguns casos também promover a obstrução venosa ${ }^{19}$.

Os infartos anêmicos ou brancos ocorrem em órgãos sólidos como o coração, rins e pâncreas, resultantes da oclusão de artérias. Os do tipo hemorrágicos ou vermelhos ocorrem em tecidos frouxos e de circulação dupla como o pulmão e intestino. Correspondem a áreas localizadas de necrose com hemorragia maciça associada $^{18}$.

O infarto do miocárdio ou ataque cardíaco é considerado a forma mais importante de cardiopatia isquêmica21. É responsável pelo maior número de mortes nos Estados Unidos e semelhantemente possui papel preponderante nos indicadores de morbimortalidade no Brasil $^{22}$.

Decorre da ausência prolongada de aporte sanguíneo para o músculo cardíaco e pode ser classificado como um tipo de choque cardiogênico, no qual ocorre uma falência da bomba miocárdica e, devido ao dano intrínseco, uma diminuição acentuada do débito cardíaco ${ }^{5,18,19}$.

Outra importante patologia cardiovascular, o acidente vascular cerebral (AVC) ocorre devido à ruptura e consequentemente sangramento na região cerebral, ou por trombose de uma das artérias principais que irrigam o cérebro. Em qualquer dos casos leva a perda de irrigação local, podendo haver comprometimento das funções e dano neurológico severo a depender da região afetada'.

Diversas são as estratégias farmacológicas para - tratamento das patologias cardiovasculares de origem tromboembólica. Os anticoagulantes orais e injetáveis, bem como os antiagregantes plaquetários, são amplamente utilizados como medida profilática ou terapêutica para trombose de origem venosa e arterial, respectivamente. Já os vários fármacos fibrinolíticos são usados principalmente para 
dissolver coágulos já formados e restabelecer a circulação de pacientes internos em ambiente hospitalar $9,10,17$.

Há mais de 50 anos, a varfarina vem sendo o fármaco de escolha para a terapia anticoagulante a longo prazo ${ }^{23,24}$. É $O$ anticoagulante mais importante e mais utilizado no mundo e comumente prescrito para profilaxia ou tratamento da doença tromboembólica venosa ${ }^{2,25,26}$.

Trata-se de um derivado cumarínico, antagonista da vitamina $K$, que interfere na síntese dos fatores de coagulação vitamina $K$ dependentes, que incluem protrombina (fator II), fatores VII, IX e X, consequentemente interferindo drasticamente na cascata da coagulação 27,28 .

A terapia anticoagulante com varfarina possui uma estreita janela terapêutica, sendo necessário acompanhamento contínuo dos níveis de coagulação, a fim de evitar, principalmente, episódios hemorrágicos espontâneos. O efeito da varfarina é monitorado através da dosagem do tempo de protrombina (TP), que é expresso como um Índice Internacional Normalizado (INR) ${ }^{26}$.

Os testes de coagulação sanguínea incluindo o tempo de sangramento (TS) e tempo de coagulação (TC) não fornecem efetivos resultados para pacientes que utilizam essa terapia anticoagulante, pois o teste do TS pode se prolongar, de forma especial, principalmente na ausência de plaquetas e não na ausência de qualquer um dos fatores de coagulação'.

O teste de TC também é ineficaz devido a uma variabilidade de tempo obtido a depender do método utilizado. Em substituição a este a dosagem dos próprios fatores de coagulação fornecem melhores resultados, como o teste do TP'.

Diante desta variabilidade foi instituído em 1983 - INR, um sistema uniforme para padronizar qual melhor conduta a seguir para $\circ$ controle dos níveis dos fatores de coagulação em pacientes anticoagulados. O INR representa um valor numérico a partir da relação entre o TP do paciente e a média do intervalo normal da coagulação, corrigido este valor através da multiplicação pelo Índice de Sensibilidade Internacional (ISI) ${ }^{26}$.
O INR ideal é igual a 1.0, sendo quanto maior esse valor, menor é a coagulação sanguínea. $O$ intervalo terapêutico ideal para um paciente em terapia anticoagulante está entre $2 \cdot 0-3 \cdot 5^{26,28}$.

A heparina é um anticoagulante injetável que inibe a coagulação por meio da ativação da antitrombina III que inibe a trombina e outras serinaproteases por ligação ao sítio ativo de serina. A heparina modifica esta interação ligando-se à antitrombina III, alterando sua conformação e acelerando sua velocidade de ação, impedindo desta forma as etapas finais da via comum da cascata da coagulação ${ }^{10}$.

A administração pode ser por via intravenosa ou subcutânea, devido a sua carga e grande tamanho ${ }^{29}$. As heparinas têm ação imediata, enquanto os anticoagulantes orais levam vários dias para exercer e terminar seu efeito, sendo as heparinas geralmente utilizadas na fase aguda da doença ${ }^{2,28}$.

A hemorragia também constitui a principal complicação do uso da heparina e pode ocorrer mesmo com monitorização adequada dos parâmetros da coagulação. Esse controle pode ser através da realização do exame do tempo de tromboplastina ativada (TTPA) ou TC, que é menos sensível. Além disso, consideram-se necessárias a determinação prévia do TP e a contagem de plaquetas ${ }^{10}$.

Como as plaquetas desempenham importante papel na formação do coágulo não é surpresa que os antiagregantes plaquetários tenham grande valor terapêutico no tratamento da doença tromboembólica, principalmente na de origem arterial $^{2}$.

O ácido acetilsalicílico (AAS), principal representante deste grupo, além dos seus efeitos analgésico, antitérmico e anti-inflamatório amplamente conhecidos possui excelente efeito antitrombótico quando administrado em baixas doses ${ }^{10,14}$. Com posologia simples, custo reduzido e eficácia comprovada, possui indicação quase universal diante de quadros de infarto agudo do miocário ${ }^{21,22}$.

Essa droga é um inibidor irreversível da enzima cicloxigenase, bloqueando a produção de tromboxano A2, fundamental para o recrutamento e ativação de mais plaquetas e essencial para 
a formação do coágulo. No momento em que plaquetas não sintetizam novas enzimas, a ação do ácido acetilsalicílico na cicloxigenase plaquetária, e consequentemente na função, é permanente, persistindo durante toda a vida plaquetária (sete a dez dias) e restabelecida só após uma renovação sequencial ${ }^{14}$.

Outros antiplaquetários estão disponíveis no mercado nacional, mas a sua utilização clínica é bem menor principalmente em decorrência do custo destas substâncias. Como coadjuvante ou alternativa ao AAS, a droga mais utilizada é o Clopidogrel. Trata-se de um fármaco antagonista de receptores plaquetários (tipo P2Y2) dotado de ação de menor duração quando comparado ao AAS.

Embora a varfarina, as heparinas e o AAS sejam efetivos na prevenção da formação e propagação de trombos, esses fármacos, em geral, são ineficazes contra coágulos preexistentes. Para estes casos, se faz necessário o uso, em ambiente hospitalar, de agentes trombolíticos ou fibrinolíticos capazes de dissolver coágulos já formados, desobstruindo o vaso, restabelecendo o fluxo sanguíneo e prevenindo a necrose tecidual ${ }^{3}$.

Dentre os fármacos fibrinolíticos os mais frequentemente utilizados são a estreptoquinase, alteplase e tenecteplase. A estreptoquinase é uma proteína que ativa o plasminogênio. É administrada por via endovenosa e associada ao AAS desenvolve amplo efeito benéfico na redução da mortalidade em casos de infarto agudo do miocárdio. Alteplase e tenecteplase são t-PAs recombinantes mais ativas sobre o plasminogênio ligado a fibrina do que sobre o plasminogênio plasmático e, portanto, são consideradas seletivas para o coágulo2.

Conforme descrito, as indicações da terapia antitrombótica vêm se tornando cada vez mais frequentes, devido à alta prevalência de doenças cardiovasculares de origem tromboembólica, consequentemente aumentando a ocorrência de pacientes usuários deste tipo de medicação nos consultórios odontológicos ${ }^{2,12,26}$.

A utilização de algum destes fármacos, mesmo que para fins terapêuticos, altera os mecanismos normais da hemostasia, retardando o processo de coagulação, podendo acarretar em acidentes ou complicações durante o atendimento ${ }^{21}$. A hemorragia trans ou pós-operatória é uma das complicações mais temidas na prática odontológica ${ }^{14,30}$.

A conduta clássica para reduzir o risco hemorrágico de usuários de AAS baseava-se na interrupção da medicação entre cinco a sete dias antes de cirurgias eletivas, diante da condição do fármaco de inibidor irreversível da enzima cicloxigenase ${ }^{14}$.

No entanto, Ardekian et al (2000) apud Kreisner et $\mathrm{al}^{31}$ verificaram em seu estudo que de maneira similar os grupos controle e experimental, que interromperam a terapia com AAS e que continuaram utilizando a droga, respectivamente, apresentaram tempo de sangramento dentro dos parâmetros normais diante de procedimentos cirúrgicos de complexidade equivalente. Fato este podendo ser justificado pela existência de indivíduos mais sensíveis ou não aos efeitos da medicação, ou ainda devido a velocidades variáveis de metabolização do fármaco ${ }^{14}$.

Em se tratando de anticoagulantes orais, classicamente recomendavase que os pacientes interrompessem a medicação por quatro a cinco dias antes de uma cirurgia eletiva e se a condição clínica exigisse, esta poderia ser substituída pela heparina por possuir ação rápida, meia-vida mais curta e antagonismo bastante eficaz com a utilização de sulfato de protamina, podendo a heparina ser suspensa até mesmo horas antes do procedimento a fim de evitar episódios hemorrágicos ${ }^{14}$.

No entanto, verifica-se que as complicações tromboembólicas apresentam uma probabilidade três vezes superior de ocorrer em pacientes que interrompem a terapia em detrimento das complicações hemorrágicas em pacientes sem que haja interrupção da terapia ${ }^{30}$.

$\mathrm{Na}$ manutenção da terapia, interrupção, ou apenas a redução da posologia, em nenhum desses esquemas há isenção de riscos, sendo estritamente necessário antes de se optar por algum dos mesmos uma avaliação completa da condição sistêmica do paciente, acompanhamento do grau de anticoagulação através de exames laboratoriais, principalmente INR para pacientes usuários de anticoagulantes, e da classificação do trauma envolvido no procedimento odontológico a 
ser realizado ${ }^{12}$.

Kudsi ${ }^{25}$ exemplifica situações de baixo e alto risco cirúrgico, onde considera exodontias simples de um único dente ou menos de quatro dentes como de baixo risco cirúrgico, exodontias complicadas, exodontias múltiplas de mais de cinco elementos, biópsias e instalação de implantes como de alto risco.

Sugerem-se valores de INR considerados apropriados para a realização de alguns procedimentos. Para situações onde é esperado uma hemorragia mínima ou exodontia de um único dente é indicado INR até 4.0, em casos de hemorragia moderada, exodontias múltiplas ou de terceiros molares é aceitável INR até 3,5 , em situações onde se espera uma maior perda sanguínea $\circ$ INR não deve ultrapassar 3.0 e não deve ser realizado qualquer tipo de procedimento diante de INR superior a 5.026 .

Dantas et $\mathrm{al}^{12}$ analisaram a ocorrência de complicações hemorrágicas em pacientes usuários de fármacos anticoagulantes submetidos a exodontias consideradas de pequeno e médio trauma, onde verificaram que mesmo mantendo-se a medicação nestes pacientes, quando associados a técnicas cirúrgicas atraumáticas e correta avaliação da condição de hipocoagulação através do INR, foi possível a realização com segurança.

Araújo et $a^{26}{ }^{26}$ relataram a realização de exodontias, respeitando todas as etapas recomendadas e sem modificação na terapêutica anticoagulante, verificando adequada realização dos procedimentos sem intercorrências e de forma satisfatória, sendo necessário somente utilizar métodos e medidas hemostáticas locais (Figura 3) em um dos casos.

Murphy et $\mathrm{al}^{33}$ a partir da aplicação de um questionário para cirurgiões-dentistas verificaram uma variedade de práticas na abordagem de pacientes em terapia anticoagulante e antiplaquetária, evidenciando um manejo aleatório. Nesta pesquisa ${ }^{33}, 25 \%$ dos profissionais, apesar de realizarem procedimentos odontológicos em pacientes anticoagulados, nunca utilizaram $\circ$ INR como exame complementar pré-operatório, da mesma forma com o paciente que faz uso de antiagregante plaquetário, onde $95 \%$ dos dentistas interrompem às vezes ou sempre a medicação.

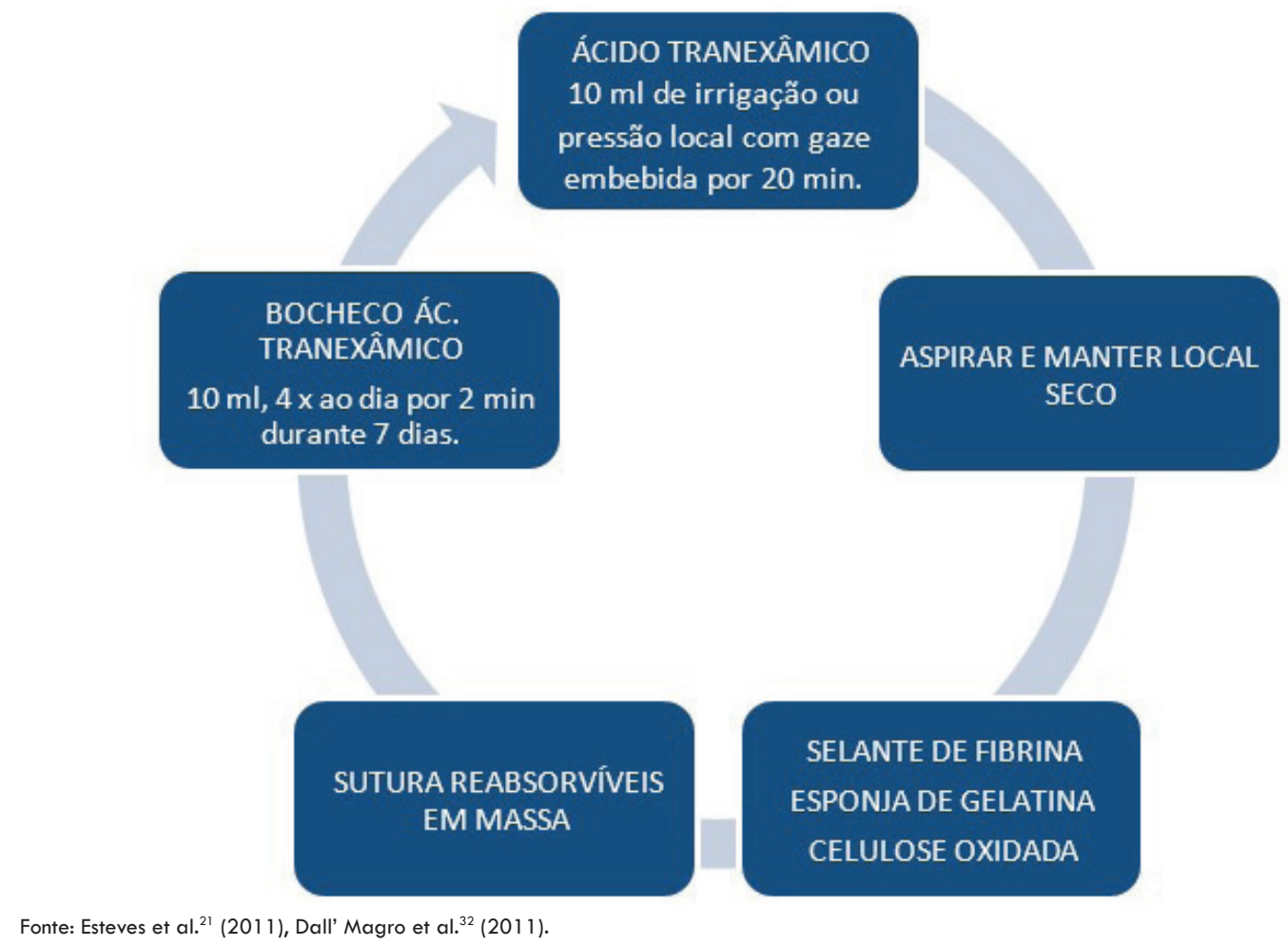

Figura 3. Métodos e medidas de hemostasia local. 
Por isso, a tendência atual é que pacientes que fazem uso de anticoagulantes orais possam se submeter a procedimentos odontológicos, sem necessidade de qualquer interrupção ou modificação na terapia, mas com ênfase em medidas preventivas de hemostasia local $^{21,26}$.

Este fato está associado principalmente devido a hemorragia imediata que acontece em qualquer cirurgia oral, ocorrer de modo semelhante em pacientes hipocoagulados ou não. Pois, geralmente, ocorre somente a ruptura de vasos de pequeno calibre em que a hemostasia depende principalmente da fase plaquetária da coagulação, portanto não sendo alterada pelos fármacos anticoagulantes ${ }^{30}$.

Os estudos apontam que a descontinuação da terapia anticoagulante deve ser evitada sempre que os exames clínicos e laboratoriais permitam a execução do procedimento sem a suspensão do medicamento. Porém, nas situações em que a avaliação da condição sistêmica do paciente indica a suspensão ou alteração da medicação, o médico cardiologista deve ser consultado ${ }^{21}$.

No entanto, ainda é controversa a abordagem ao paciente que faz uso de antiagregante plaquetário, considerando que a conduta de interromper o uso, no mínimo, cinco dias antes do procedimento é ainda considerada prudente para reduzir o risco de hemorragia, e diferentemente dos anticoagulantes, aparentemente sem prejuízos ao paciente ${ }^{14}$.

Diante desta variabilidade de condutas, o planejamento para $\circ$ atendimento de pacientes em terapia antitrombótica deve estar baseado em coletas de dados e planejamento durante a etapa pré-operatória e a seguir a correta execução, durante as etapas trans-operatória e pós-operatória (Figura 4).

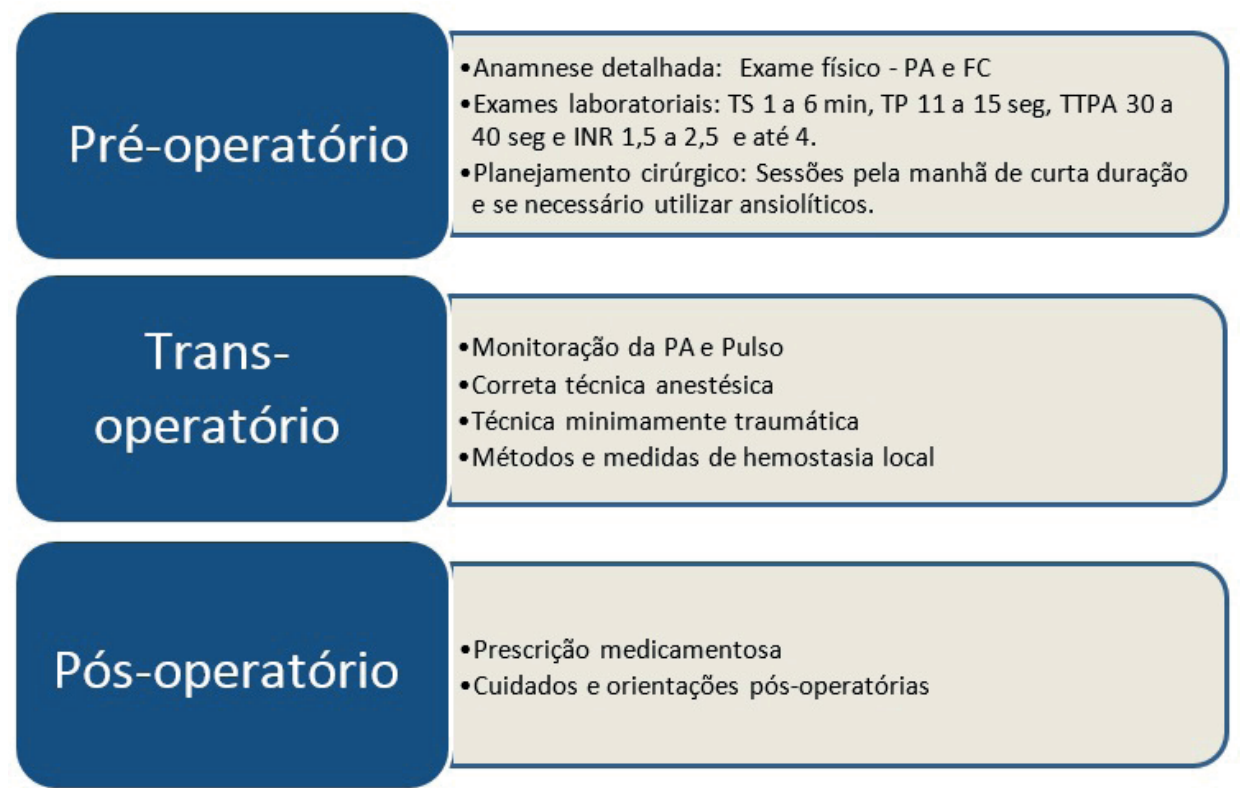

Legenda: PA- Pressão Arterial; FC- Frequência cardíaca; TS- Tempo de Sangramento; TP- Tempo de protrombina; TTPA- Tempo de tromboplastina ativada; INR- Índice Internacional Normalizado.

Fonte: Esteves et al..$^{21}(2011)$, Alencar et al. ${ }^{34}(2011)$, Toscano et al. ${ }^{28}$ (2009).

Figura 4. Cuidados pré, trans e pós-operatórios para pacientes em terapia antitrombótica

$\mathrm{Na}$ literatura ainda são escassas informações a respeito da utilização de fibrinolíticos por pacientes que necessitam se submeter a atendimento odontológico eletivo, devido principalmente pela utilização destes somente por pacientes hospitalizados. Outra hipótese é que tradicionalmente preconiza-se o protocolo de espera de seis meses para a execução de outros procedimentos cirúrgicos não-cardíacos em pacientes infartados, por exemplo, o que já justificaria a utilização de outra terapia, anticoagulante ou antiplaquetária, durante referido período. 


\section{CONSIDERAÇÕES FINAIS}

A tendência atual é que pacientes que fazem uso de anticoagulantes orais possam se submeter a procedimentos odontológicos, sem necessidade de qualquer interrupção ou modificação na terapia, mas com ênfase em medidas preventivas de hemostasia local. Ainda é controversa a abordagem ao paciente que faz uso de AAS, considerando que a conduta de interromper $O$ uso dias antes do procedimento continua sendo prudente para reduzir $\circ$ risco de hemorragia, e diferentemente dos anticoagulantes, aparentemente sem prejuízos ao paciente.

Os anticoagulantes orais e injetáveis, bem como os antiagregantes plaquetários, são amplamente utilizados como medida profilática e terapêutica de uma das mais prevalentes doenças cardiovasculares, a doença trombótica. Estima-se que mais de $30 \%$ da população acima de 70 anos apresenta indicação desta terapêutica, consequentemente aumentado a ocorrência de pacientes usuários deste tipo de terapia no consultório odontológico.

O cirurgião-dentista deve estar apto para - atendimento destes pacientes, realizando procedimentos de forma segura, prevenindo complicações hemorrágicas, baseado em um planejamento individualizado através de minuciosa coleta de dados, anamnese, exames complementares, planejamento pré-operatório e a seguir a correta execução das etapas transoperatória e pósoperatória.

\section{REFERÊNCIAS}

1. Guyton AC, Hall JE. Tratado de Fisiologia Médica. $11^{\mathrm{a}}$ ed. Rio de Janeiro:

Elsevier; 2006. P.457-68

2. Rang HP, Dale MM, Ritter JM, Flower RJ, Henderson G. Rang \& Dale: farmacologia. $7^{a}$ ed. Rio de Janeiro: Elsevier; 201. P. 294-307

3. Golan DE, Tashiian AH Jr, Armstrong EJ, Armstrong AW. Princípios de Farmacologia: a base fisiopatológica da farmacoterapia. $2^{a}$ ed. Rio de Janeiro: Guanabara
Koogan; 2012. P.356

4. Metze K. Distúrbios da Circulação. In: Brasileiro Filho G. Bogliolo, Patologia. $7^{a}$ ed. Rio de Janeiro: Guanabara Koogan; 2006. P.97-119

5. Teixeira CS, Pasternak B Jr, Silva-Sousa YTC, Perez DEC. Tratamento odontológico em pacientes com comprometimento cardiovascular. Revista Sul-Brasileira de Odontologia. 2008;5(1):68-76

6. Pelegrino FM, Dantas RAS, Corbi ISA, Carvalho ARS, Perfil sócio demográfico e clínico de pacientes em uso de anticoagulantes orais. Rev Gaúcha Enferm. 2010;31(1):123-8

7. The World Health Organization. Cardiovascular diseases [Internet]. 2009 [acesso 10 jun 2013]. Disponível em http://www.who.int/mediacentre/factsheets/fs317/en/ index.html

8. Weitz Jl. Coagulação sanguínea e fármacos anticoagulantes, fibrinolíticos e antiplaquetários. In: Brunton LL, Chabner BA, Knollmann BC. As bases farmacológicas da terapêutica de Goodman \& Gilman. $12^{a}$ Ed. Porto Alegre: Artmed; 2012. P. 849-74

9. Silverthorn DU. Fisiologia humana: um abordagem integrada. $5^{a}$ ed. Porto Alegre: Artmed; 2010. P.55864

10. Pereira MG. Anticoagulantes, antiagregantes plaquetários e trombolíticos. In: Silva P. Farmacologia. $8^{a}$ ed. Rio de Janeiro: Guanabara Koogan; 2012. P.588-602

11. Brasil. Ministério da Saúde. Secretaria de Vigilância em Saúde. Sistema

Nacional de Vigilância em Saúde: Relatório de Situação: Alagoas. Brasília. DF. 2011

12. Dantas AK, Deboni $M C Z$, Piratininga JL. Cirurgias odontológicas em usuários de anticoagulantes orais. Revista Brasileira de Hematologia e Hemoterapia. 2009;31:337-40

13. Cavalheiro-Filho C, Rached RA. Reversão da anticoagulação oral em situações de risco. Rev Soc Cardiol Estado de São Paulo. 2006; 16(4):287294

14. Kreisner PE, Ferreira AGM, Heitz C, Woitchunas GFP, Martins CAM. Procedimentos cirúrgicos em pacientes que fazem uso de ácido acetilsalicílico ou anticoagulantes orais. Revista da Faculdade de 
15. Wahl MJ. Myths of dental surgery in patients receiving anticoagulante therapy. J Am Dent Assoc. 2000; $131(3) ; 77-81$

16. Franchini KG. Circulação arterial e hemodinâmica: física dos vasos sanguíneos e da circulação. In: Aires MM. Fisiologia. $4^{a}$ ed. Rio de Janeiro: Guanabara Koogan; 2012 . P.487- 488

17. Tortora GJ, Derrickson B. Princípios de Anatomia e Fisiologia. $12^{a}$ ed. Rio de Janeiro: Guanabara Koogan; 2010. P.695-700

18. Mitchell RN, Kumar V, Abbas AK, Fausto N. Robbins \& Cotran - Fundamentos de Patologia: bases patológicas das doenças. $7^{a}$ ed. Rio de Janeiro: Elsevier; 2006. P.77-90

19. Almeida PC, Barretto Netto M, Montenegro MR. Perturbações circulatórias. In: Franco M, Montenegro MR, Brito Thales de, Bacchi CE, Almeida PC de. Patologia: processos gerais. $5^{a}$ ed. São Paulo: Atheneu Editora; 2010. P. 126-33

20. Barros-Sena MA, Genestra M. Profilaxia da trombose venosa profunda em pós-operatório de cirurgias ortopédicas em um hospital de traumatoortopedia. Revista Brasileira de hematologia e hemoterapia. 2008;30(1):29-35

21. Esteves JC, Simão FB, Ricieri CB, Fattah CMRS, Santos PSS, Aranega AM. Assistência cirúrgicoodontológica a pacientes com história de infarto do miocárdio. Rev Gaúcha Odontol. 201 1;59(2):285-91

22. Escosteguy CC, Portela MC, Medronho RA, Vasconcellos MTL. Infarto agudo do miocárdio: perfil clínico-epidemiológico e fatores associados ao óbito hospitalar no município do Rio de Janeiro. Arq Bras Cardiol. 2003;80(6):593-99

23. Karsh ED, Erdogan Ö, Esen E, Acartürk E. Comparasion oh the Effects of Warfarin and Heparin on Bleeding Caused by Dental Extraction: A Clinical Study. J Oral Maxilofac Surg. 201 1;69:2500-07

24. Leiria TLL, Pellanda L, Miglioranza MH, Sant'Anna RT, Becker LS, Magalhães E, et al. Varfarina e Femprocumona: experiência de um ambulatório de anticoagulação. Arq Bras Cardiol. 2010;94(1):41-45

25. Kudsi Z, Dalati MHN, Sibai L, Koussayer.

Management of Bleeding Disorders em the Dental
Update. $2012 ; 39: 358-363$

26. Araujo FM, Lopes MVN, López AR, López AG, Souza LN. Cirurgia Oral em Pacientes sob Terapia com Anticoagulantes Orais: Revisão da Literatura e Apresentação de Dois Casos Clínicos. Rev Port de Estomatologia, Medicina Dentária e Cirurgia Maxilofacial. 2010;51(4):219-224

27. Holtz EMA. Relação da doença periodontal com a anticoagulação oral e os benefícios do tratamento periodontal em pacientes anticoagulados [Dissertação]. Rio de Janeiro (RJ): Universidade do Estado do Rio de Janeiro; 2009

28. Toscano NJ, Holtzclaw DJ, Moss HD, Shumaker N. Implant Considerations in the Anticoagulated Patient: A Review. Titanium. 2009; 1:52-59

29. Cerveró AJ, Roda RP, Bagán JV, Soriano YJ. Dental treatment of patients with coagulation factor alterations: An update. Med Oral Patol Oral Cir Bucal. 2007; 1 2:E380-7

30. Marques MS, Almeida AP, Lopes MG. A terapêutica anticoagulante em cirurgia oral - revisão de literatura. Rev Port Estomatol Cir Maxilofac. 2005;46(1):31-6

31. Ardekian L, Gaspar R, Peled M et al. Does Low-dose aspirin therapy complicate oral surgical procedures?. JADA. 2000; 131:331-35 apud Kreisner PE, Ferreira AGM, Heitz C, Woitchunas GFP, Martins CAM. Procedimentos cirúrgicos em pacientes que fazem uso de ácido acetilsalicílico ou anticoagulantes orais. Revista da Faculdade de Odontologia. 2003; 8(1):22-7

32. Dall' Magro AK, Ribeiro AA, Shenkel A, Samuelsson M, Studzinski MS, Almeida D. Manejo odontológico de pacientes com coagulopatias - revisão de literatura e relato de caso: síndrome de Bernard Soulier. RFO Passo Fundo. 2011 ; $16(2): 193-99$

33. Murphy J, Twohig E, McWilliams SR. Dentists' approach to patients on antiplatelet agents and warfarin: a survey of pratice. Journal of the Irish Dental Association. 2009;56(1):28-33

34. Alencar CRB, Andrade FJP, Catão MHCV. Cirurgia oral em pacientes idosos: considerações clínicas, cirúrgicas, e avaliação de riscos. Revista Sul-Brasileira de Odontologia. $2011 ; 8(2): 200-10$ 